\title{
La simulación clínica: Un aporte para la enseñanza y aprendizaje en el área de obstetricia
}

\author{
Clinical simulation: a contribution to teaching and learning in the Obstetrics area
}

\section{Simulação clínica: uma contribuição para o ensino e aprendizagem na área de obstetrícia}

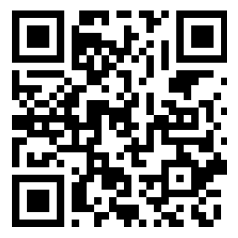

\author{
Janet Elizabeth Altamirano-Droguett \\ Universidad de Concepción \\ Concepción, Chile \\ janetaltamirano@udec.cl \\ http://orcid.org/0000-0002-8051-9034
}

\begin{abstract}
Recibido • Received • Recebido: 10 / 07 / 2017
Corregido • Revised • Revisado: 25 / 09 / 2018

Aceptado • Accepted • Aprovado: 22/ 02 / 2019
\end{abstract}

\begin{abstract}
Resumen: Este estudio de tipo descriptivo plantea una revisión bibliográfica para determinar el aporte de los tipos de simulación clínica: entrenamiento con pacientes que se simulan, juego de roles y simulaciones de paciente completo, en el aprendizaje de las matronas y matrones en formación. Se realizó una búsqueda exhaustiva de esta temática en revistas científicas indexadas Scielo y Scopus, a través del buscador Google académico y PubMed, en el contexto internacional y nacional, donde se encontró escasez de investigaciones de esta técnica didáctica en el área de obstetricia. El foco de análisis y discusión es el aprendizaje, con el fin de especificar desde una perspectiva experiencial, psicocultural y pedagógica, el valor de la simulación clínica en el desarrollo de competencias genéricas y profesionales. Finalmente, este estudio concluyó que la simulación clínica es necesaria para mejorar la seguridad y confianza del estudiantado de la carrera de obstetricia y puericultura al enfrentar sus prácticas profesionales con pacientes reales, pues, si bien se ha descrito el desarrollo de diversos tipos de aprendizajes con el uso de esta técnica, aún falta potenciar esta herramienta de enseñanza en el proceso formativo de pregrado, con el personal docente como facilitador del aprendizaje.
\end{abstract}

Palabras claves: Aprendizaje; enseñanza; obstetricia; simulación clínica.

Abstract: This descriptive study presents a bibliographic review to determine the contribution of the types of clinical simulation: simulated patient training, role play, and full patient simulators in the learning of midwives and midwives in training. An exhaustive search of this topic was carried out in Scielo and Scopus indexed scientific journals; the Google scholar search engine and PubMed, in the international and national context, were used. The search showed a lack of research on this didactic technique in the area of Obstetrics. The focus of analysis and discussion is learning, in order to specify the value of clinical simulation in the development of generic and professional competences from an experiential, psychocultural and pedagogical perspective. Finally, this study concluded that clinical simulation is necessary to improve the safety and confidence of the students enrolled in 
doi: http://dx.doi.org/10.15359/ree.23-2.9

URL: http://www.una.ac.cr/educare

CORREO: educare@una.cr

the Obstetrics and Child Care programs when facing their professional practices with real patients. Even though the development of different types of learning has been described with the use of this technique, this teaching tool needs to be strengthened in the undergraduate training process, with the teaching staff as a facilitator of learning.

Keywords: Learning; teaching; obstetrics; clinical simulation.

Resumo: Este estudo descritivo apresenta uma revisão da literatura para determinar a contribuição dos tipos de simulação clínica: treinamento simulado de pacientes, role-playing e simulações de paciente completo, na aprendizagem das matronas e matrones em formação. Realizou-se uma busca exaustiva desta temática em revistas científicas indexadas Scielo e Scopus, através do buscador Google acadêmico e PubMed, em contexto internacional e nacional, onde se encontrou escassez de investigações desta técnica didática na área de obstetrícia. O foco de análise e discussão é a aprendizagem, com o fim de especificar desde uma perspectiva experiencial, psicocultural e pedagógica, o valor da simulação clínica no desenvolvimento de concorrências genéricas e profissionais. Finalmente, este estudo concluiu que a simulação clínica é necessária para melhorar a segurança e confiança do estudante da carreira de obstetrícia e puericultura ao enfrentar suas práticas profissionais com pacientes reais, pois, apesar de descrever o desenvolvimento de diversos tipos de aprendizagens com o uso desta técnica, ainda falta potenciar esta ferramenta de ensino no processo formativo de pré universitário, com o pessoal docente como facilitador da aprendizagem.

Palavras-chave: Aprendizagem; ensino; obstetrícia; simulação clínica.

\section{Introducción}

Actualmente, el paradigma educativo está centrado en el estudiantado y, para que el aprendizaje sea efectivo, se ha requerido que las instituciones de educación superior renueven las metodologías de enseñanza que han sido por décadas tradicionales, para cambiarlas por otras innovadoras, dentro de las cuales se encuentra la simulación clínica. Esta metodología ha sido definida como una técnica, no una tecnología, para sustituir o ampliar las experiencias reales a través de experiencias guiadas, que evocan o replican aspectos sustanciales del mundo real, de una forma totalmente interactiva (Gaba, 2004).

A la fecha, existen escasos estudios de simulación clínica en el área disciplinar de la obstetricia, por lo tanto, plantear una revisión bibliográfica sobre esta didáctica es pertinente, con el objetivo de determinar el aporte de los tipos de simulación clínica, entrenamiento con personas pacientes simuladas, juego de roles y simuladores de paciente completo en el aprendizaje profesional de la matrona y el matrón. Su relevancia radica en que la simulación clínica contribuye con la calidad y seguridad en la atención de pacientes, pues permite que el estudiantado adquiriera destrezas y confianza en sí mismo, antes de enfrentar situaciones reales (Urra, Sandoval e Irribarren, 2017). 
Para elaborar esta revisión, se realizó una búsqueda exhaustiva de artículos en revistas de ciencias de la salud indexadas en Scielo y Scopus, a través del buscador digital google académico y PubMed. Además, se utilizaron las palabras claves: aprendizaje; enseñanza; obstetricia; simulación clínica; y el título del artículo en el contexto internacional y nacional. También, se accedió a tesis y textos relacionados con los contenidos específicos de este estudio vía online y, en formato físico.

Este artículo se inicia con algunos planteamientos teóricos del modelo de competencias y perspectivas psicoculturales y pedagógicas del aprendizaje que sustentan la simulación clínica. Posteriormente, describimos los tres tipos de simulación clínica seleccionados, ejemplificándolos desde la experiencia profesional y destacando las competencias genéricas y específicas que se logran con el apoyo en cada uno de ellos. Finalmente se identifican los desafíos futuros de esta metodología de enseñanza en beneficio no solo de la matronería, sino también, de todas las profesiones del área de la salud y de otras carreras afines, que puedan replicar esta experiencia docente en las aulas universitarias.

\section{La educación del siglo XXI}

En los comienzos del nuevo siglo, se ha observado una demanda de educación superior sin precedentes, acompañada de una gran diversificación y una mayor toma de conciencia de la importancia fundamental que el nivel terciario reviste para el desarrollo sociocultural, económico y para la construcción del futuro, por lo cual, las nuevas generaciones deberán estar preparadas con nuevas competencias (Altamirano, 2011).

En el marco del Espacio Europeo de Educación Superior y con la Declaración de Bolonia (1999), se han implementado, en las universidades, nuevos modelos de enseñanza y evaluación que centran su formación en el estudiantado (Arriazu, 2013). Esto es posible con la inclusión de competencias, la introducción de nuevas metodologías docentes y de sistemas evaluativos acordes a las didácticas (Riesco, 2008).

En este contexto, surge el Proyecto Tuning (Beneitone et al., 2007), desarrollado en la Unión Europea, que planteó un nuevo modelo formativo que concibe los resultados del aprendizaje como desempeños observables, y definió que las estructuras educativas y el contenido son tareas fundamentales de las instituciones de educación superior. Esta misma experiencia se replicó entre los años 2004 y 2007 en América Latina, impulsando la discusión respecto a la calidad de la educación terciaria.

Es así como emerge un nuevo modelo educativo basado en competencias. Su efectividad se logra a través del uso de metodologías innovadoras como la simulación clínica, que asegura el desarrollo de las dimensiones cognitivas, procedimentales y actitudinales dentro de un contexto social a lo largo de la vida. 
doi: http://dx.doi.org/10.15359/ree.23-2.9

URL: http://www.una.ac.cr/educare

CORREO: educare@una.cr

\section{Las competencias en el área de la obstetricia}

Cuando hablamos de competencias, se deben tomar de referencia los cuatro pilares básicos del saber: "Aprender a conocer, aprender a hacer, aprender a vivir juntos, aprender a ser" (Delors, 1996, p. 9), que sustentan las dimensiones en el desarrollo personal y profesional de quien se dedica a la matronería.

Una competencia es un "saber hacer" complejo, que se manifiesta en la actuación efectiva sobre una situación problemática, para cuya solución se movilizan integradamente diferentes habilidades, conocimientos y actitudes (Camargo-Escobar y Pardo-Adames, 2008; Díaz-Barriga, 2005; Rodríguez, 2007; Yaniz y Villardón, 2006). Las competencias genéricas o transversales se refieren al desarrollo de las personas, tanto en su dimensión intrapersonal como de interacción con otras (Ramírez y Medina, 2008). Las competencias específicas reflejan el desempeño propio de cada profesión (Hawes y Corvalán, 2005).

Estas competencias dan origen a un modelo educativo enfocado en el estudiantado, el cual permite converger sus habilidades cognoscitivas, psicológicas, sensoriales, motoras y afectivas. Por lo tanto, el aprendizaje debe potenciar la integración de las disciplinas del conocimiento, las habilidades genéricas y la comunicación de ideas (Argudín, 2001); y la enseñanza debe enfocarse en los saberes (conocimientos), en el control de las interacciones sociales y en la respuesta frente a las personas (Ortega, 2008).

Las universidades asumen este nuevo paradigma educativoy, progresivamente, las carreras del área de la salud han ido renovando sus currículos de pregrado. Dentro de este contexto, las carreras de Obstetricia y Puericultura pertenecientes al Consejo de Rectores de las Universidades Chilenas (CRUCH) han ido adoptando estos nuevos requerimientos pedagógicos, definiendo sus perfiles de egreso con base en competencias genéricas y específicas, e incluyendo el uso de nuevas metodologías innovadoras, como la simulación clínica.

A partir de lo anterior, seleccionamos las competencias más desarrolladas con la simulación clínica en el área de la obstetricia, las generales son: comunicación, pensamiento crítico, toma de decisiones, trabajo en equipo, consistencia ética; y las competencias profesionales: 1) Atención integral a la mujer durante los períodos del embarazo, parto y puerperio normal. 2) Pesquisa y derivación oportuna de la anormalidad obstétrica. 3) Actuar en forma eficiente y eficaz ante situaciones de urgencia durante el embarazo, parto y puerperio (CNA, 2007).

Todas ellas, se consolidan a lo largo de la formación de pregrado, sin embargo, el estudiantado las adquiere en la etapa preclínica, en un entorno educativo simulado, con características muy cercanas a la realidad, que le permitan enfrentar posteriormente, sus experiencias prácticas con mayor preparación, confianza y seguridad en sí mismo. 


\section{Concepto y tipos de simulación clínica}

La simulación es un método muy útil en las carreras del área de la salud, pues acelera el proceso de aprendizaje del estudiantado y mejora su familiarización con métodos de autoevaluación, al permitir la utilización del ensayo y error como un medio de retroalimentación, previo ingreso al campo clínico real. Para Gaba (2004), la simulación es una técnica para reemplazar o amplificar una experiencia real que está a menudo inmersa en lo natural, y cuya práctica simulada evoca o replica, sustancialmente, aspectos de ese mundo real, en una forma interactiva total.

Según Ziv, Wolpe, Small y Glick (2003), la simulación clínica se clasifica en cinco categorías: 1) Simuladores de uso específico y de baja tecnología (Part task trainers), que son modelos diseñados para replicar una parte del organismo, por lo que solo permiten el desarrollo de habilidades psicomotoras básicas (Maran y Glavin, 2003). 2) Personas pacientes simuladas o estandarizadas, sujetos actores entrenados que se utilizan para instrucción y evaluación de habilidades y comunicación (Levine y Swartz, 2008). Este tipo de simulación se desarrolla a través de la dramatización, juego de roles o role play. 3) Simuladores virtuales en pantalla, que permiten simular diversas situaciones e interactuar con el estudiantado a través de programas computacionales (Maran y Glavin, 2003). 4) Simuladores de tareas complejas, de alta fidelidad visual, auditiva y táctil, con los cuales se logra una representación tridimensional de un espacio anatómico (Maran y Glavin, 2003). 5) Simuladores de paciente completo, maniquíes de tamaño real, manejados computacionalmente, que simulan aspectos anatómicos y fisiológicos. Estos, permiten desarrollar competencias en el manejo de situaciones clínicas complejas y el trabajo en equipo (Lane, Slavin y Ziv, 2001).

Otro punto importante de señalar es el concepto de fidelidad de los simuladores o de una simulación. Tradicionalmente, se ha utilizado este término para definir el grado de realismo de los modelos y de la experiencia en la que se usan. Se dividen en tres niveles (Maran y Glavin, 2003): 1) Simulación de baja fidelidad, mencionados anteriormente como Part task trainers. 2) Simulación de mediana fidelidad combina el uso de una parte anatómica, con programas computacionales de menor complejidad. También están incluidos juegos de roles y pacientes que se simulan con entrenamiento. 3) Simulación de alta fidelidad se refiere a los simuladores de paciente completo o de tamaño real. Esto significa que, a medida que aumenta la fidelidad, aumenta el realismo (Velasco, 2013). Por lo tanto, los niveles de fidelidad los determina el medio en el que se desenvuelve el ejercicio, los factores asociados a los participantes (estudiantes, docentes y sujetosn actores), y los materiales utilizados (Velasco, 2013).

Por su parte, en el currículo de pregrado de Obstetricia y Puericultura, se escalan las actividades de simulación clínica según su complejidad: en los dos primeros años, se utiliza simulación de baja fidelidad, tercer año de mediana fidelidad, cuarto y quinto año, se emplea 
doi: http://dx.doi.org/10.15359/ree.23-2.9

URL: http://www.una.ac.cr/educare

CORREO: educare@una.cr

simulación de alta fidelidad; esta última se fortalece en el postgrado. Es decir, a medida que avanza el proceso formativo, se complejiza el tipo de metodología empleada. Frente a esto, nuestro análisis se focalizará en las categorías de simulación clínica: sujetos pacientes simulados o estandarizados y juego de roles o role play, ambos de mediana fidelidad, y los simuladores de paciente completo, de alta fidelidad.

\section{Enfoque psicocultural y pedagógico de la simulación clínica}

En este estudio se revisó la técnica de la simulación clínica como herramienta del aprendizaje, para definir su aporte en la formación de la matrona y del matrón.

Para comenzar, definimos el aprendizaje como un proceso de cambio relativamente permanente en el comportamiento de una persona, este es generado por la experiencia (Feldman, 2005) y supone un cambio en la capacidad conductual, que debe ser perdurable en el tiempo y es fundamental que el aprendizaje ocurra a través de la práctica (Schunk, 2005).

De acuerdo con las teorías cognoscitivas del aprendizaje, el personal docente debe potenciar el aprendizaje significativo en el estudiantado universitario, quien debe desarrollar su capacidad reflexiva y crítica, y ser más resistente al olvido (Eleizalde, Parra, Palomino, Reyna y Trujillo, 2010). Según Ausubel, Novak y Hanesion (1990), un aprendizaje significativo es cuando el alumnado relaciona los significados nuevos con conocimientos previos, de manera no arbitraria y sustancial, causando una expansión o modificación de la información de la memoria a largo plazo. La simulación clínica permite adquirir conocimientos, habilidades y actitudes frente a una situación de riesgo obstétrico. Si bien, la conducta es aprendida por cada estudiante en un contexto simulado, el hecho de vivenciar esa situación con antelación, almacena el conocimiento y, por ende, transfiere esta información a un contexto clínico real. Además, el personal docente, en el momento del debriefing (retroalimentación), propugna desde la deducción vincular la práctica con la teoría. Esto, sin duda, beneficia la enseñanza y las experiencias determinan si el alumnado encuentra significativo el aprendizaje (Schunk, 2005).

Por otra parte, Bruner (1966) señala que el aprendizaje por descubrimiento puede alcanzar un aprendizaje significativo, donde los maestros y las maestras son quienes facilitan el aprendizaje de sus estudiantes, quienes aprenden por sí. Este autor plantea que el descubrimiento es, en esencia, cuestión de reordenar o transformar las evidencias de tal modo que se pueda franquear los datos así reagrupados para obtener nuevos discernimientos (Bruner, 1961). Este tipo de aprendizaje es ventajoso en las actividades de solución de problemas, que activa la motivación del estudiantado y la adquisición de habilidades comunicacionales y psicomotoras (Schunk, 2005). En este caso, preferentemente el juego de roles y el uso de simuladores de tamaño real contribuyen en este tipo de aprendizaje. 
Por otro lado, existe la postura psicológica y filosófica denominada constructivismo, que enfatiza "las relaciones entre los individuos y las situaciones en la adquisición y el perfeccionamiento de las habilidades y los conocimientos" (Schunk, 2005, pp. 208-209). El estudiantado es un ente activo, que debe construir sus conocimientos; y el personal docente, asegura la interacción social de su alumnado a través de la didáctica. Esta interrelación involucra una acción humana conformada por herramientas y signos. Desde la perspectiva vygotskiana, "las funciones mentales y la acción humana general, están mediadas por herramientas (o "herramientas técnicas") y por signos (o"herramientas psicológicas") (Wertsch, 1993, p. 46). Esto significa que el rol del lenguaje humano es esencial para la comunicación intrapersonal. Por lo tanto, el estudiantado de obstetricia, a través de escenarios simulados con sujetos pacientes entrenados en el juego de roles o con pacientes de tamaño real, adquiere las formas verbales propias del lenguaje técnico empleado en la atención de pacientes.

Además, la simulación clínica por ser una técnica didáctica, está asociada al aprendizaje basado en problemas, que se ajusta perfectamente a las nuevas estrategias de enseñanzas a nivel de educación superior, porque no solo facilita la adquisición de competencias, sino también, promueve el trabajo en equipo, la comunicación, el liderazgo, entre otros (Alcolea-Cosín, OterQuintana, Martínez-Ortega, Sebastián-Viana y Pedraz-Marcos, 2012). Por ende, este aprendizaje tiene una base constructivista, pues el trabajo en grupos permite solucionar situaciones problemáticas que deberán enfrentar las futuras generaciones de profesionales en obstetricia.

Otro aporte de la simulación clínica en el aprendizaje se sustenta en el pensamiento de Dewey (2004), quien sostiene que las concepciones mentales deben entenderse como etapas del comportamiento del individuo que las genera. Además, los fines de la educación se originan en el medio social y no provienen del mero desarrollo de la naturaleza. De este modo, este autor propone que el aprendizaje se lleva a cabo realizando diversas actividades, en lugar de utilizar, únicamente, los tradicionales contenidos curriculares. Para ello, es relevante un proceso de compartir experiencias, a través del cual se promulga la interacción dialoguista y dialéctica, para fortalecer la reflexión en el alumnado, con el objeto de resolver un problema en diversos contextos educativos (Dewey, 2004), incluyendo los escenarios simulados.

Por lo tanto, la simulación clínica proporciona un apoyo y entrenamiento en el que se entrelazan conocimientos, habilidades y factores humanos, y utiliza herramientas activas, lenguaje técnico, escenarios simulados, pacientes con entrenamiento, infraestructura y equipamiento tecnológico que se asemeja a la realidad; todo ello favorece los diversos enfoques de aprendizajes durante la formación de pregrado de esta disciplina.

\section{La simulación clínica en el área de obstetricia}

Actualmente, en Chile y en otros países, el requerimiento de competencias clínicas esenciales para un efectivo ejercicio profesional se enfrenta con la escasez progresiva de campos 
doi: http://dx.doi.org/10.15359/ree.23-2.9

URL: http://www.una.ac.cr/educare

CORREO: educare@una.cr

clínicos donde efectuar y certificar los aprendizajes esperados. Por lo tanto, es necesario que las universidades cuenten con equipamiento e implementación acorde con esta metodología de enseñanza y con personal docente preparado que aplique, adecuadamente, los diversos tipos de simulación clínica.

Si revisamos la historia en nuestro país, la primera aproximación a la simulación se registra en la Pontificia Universidad Católica de Chile el año 2003, con una escuela de actuación, cuyo grupo actoral simulaba patologías al ser entrevistado por estudiantes de medicina. Más formalmente, en el año 2004, el Instituto DUOC UC creó el primer centro de simulación para la formación de carreras técnicas en salud, con construcción de escenarios y guías de evaluación (Escudero, Fuentes, González y Corvetto, 2016).

Desde el año 2009, las Carreras de Obstetricia y Puericultura de las Universidades de Valparaíso (Bottocio, 2016), de Chile (CHC, 2017), La Frontera (UFRO, 2012) y Concepción (Panorama Universidad de Concepción [UDEC], 2016), pertenecientes a las Instituciones de Educación Superior del $\mathrm{CRUCH}$, fueron las escuelas precursoras del sector centro sur del país en innovar sus currículos y emplear en su didáctica el uso de simulación clínica en la formación de pregrado. Para ello, han utilizado sujetos pacientes simulados y simuladores mecánicos, para potenciar las habilidades transversales (también llamadas blandas, por ejemplo, comunicación, razonamiento, trabajo en equipo) y las habilidades clínicas como técnicas (anamnesis, examen físico, ejecución de procedimientos, etc.) fundamentales en el desempeño profesional. En la zona norte, se suman las carreras de Obstetricia y Puericultura de las Universidades de Tarapacá (UTA, 2017), de Antofagasta, (Bravo, 2013) y de Atacama (UDA, 2015), que han contemplado, dentro de su innovación curricular, equipamiento, infraestructura y perfeccionamiento académico en simulación clínica, formando una masa crítica de docentes que incorporen esta metodología en sus asignaturas profesionales. Sin embargo, aún existe personal académico que mantiene una enseñanza tradicional, lo que implica resistencia al cambio de paradigma educativo. Además, en algunas de estas carreras, falta desarrollar la simulación clínica de alta fidelidad dentro del proceso formativo. Por lo tanto, estos son desafíos importantes que deben concretarse, para seguir potenciando esta metodología en beneficio de la matronería.

A continuación, se revisarán los tres tipos de simulación clínica seleccionados en el área de la obstetricia.

\section{Entrenamiento con sujetos pacientes simulados}

Un sujeto paciente simulado es una persona que no padece una enfermedad, sino que la simula o actúa para fines educativos, está capacitado para aportar a la docencia o evaluación desde el rol de paciente (Vázquez-Mata y Guillamet-Lloveras, 2009). Esta metodología presenta no solo el historial clínico del sujeto paciente, sino también, su lenguaje corporal, sus signos físicos, sus rasgos emocionales y de personalidad (Roger, 2017). 
En el área de la obstetricia, por ejemplo, este tipo de simulación clínica se realiza a través de la dramatización de una escena de una gestante que acude a un consultorio para ser atendida por la matrona, quien debe desarrollar la semiología obstétrica conformada por: entrevista, examen físico general y segmentario. Esto permite emitir una hipótesis diagnóstica con su correspondiente derivación. Previo a ello, la paciente simulada conoce el caso clínico, asume el tipo de paciente que debe caracterizar y se aprende el guion. El estudiantado que cumple con su rol profesional en la escena solamente tiene conocimiento de que asistirá a una embarazada.

El entrenamiento con sujetos pacientes simulados sirve, también, para revisar los signos y síntomas de cada enfermedad y los diversos tipos de comportamientos asumidos por diferentes pacientes. De esta manera, "el alumno se entrena en diferentes técnicas tales como el diagnóstico, la petición del consentimiento informado, la comunicación de malas noticias o el diálogo con pacientes agresivos o familiares intrusivos" (Durá, 2013, p. 75). Con este tipo de simulación se desarrollan las competencias de comunicación, pensamiento crítico, toma de decisiones, consistencia ética, y las competencias asistenciales obstétricas relacionadas con la atención integral de una mujer con patologías o sin estas en su proceso reproductivo y, en caso de descompensación, se debe actuar en forma eficiente y eficaz ante la emergencia.

Es importante señalar que, al final de cada escena, la evaluación es uno de los elementos clave del proceso de enseñanza aprendizaje, por el volumen de información que facilita al profesorado y por las consecuencias que tiene para docentes, alumnado, sistema educativo en que está integrado y la sociedad (Rodríguez, 2002). "La evaluación debe realizarse siempre y para que sea correcta, la simulación ha de tener criterios de validez y reproducibilidad para asegurar que cada grupo entrena las mismas competencias" (Palés y Gomar, 2010, p. 163).

Finalmente, si bien se requiere de un tiempo para la selección y entrenamiento de este tipo de "sujetos pacientes actores", se logra un aprendizaje significativo por el estudiantado al verse enfrentado ante escenarios similares a un recinto hospitalario.

\section{Juego de roles o role play}

Actualmente, los juegos de roles se consideran como una nueva dimensión necesaria en la formación de pregrado y postgrado."En ella, el estudiante debe desempeñarse como si estuviera ejerciendo [su] rol profesional dentro de un contexto laboral" (UDLA, 2017, p. 1). A través de este tipo de simulación se aprende a manejar situaciones clínicas complejas, solucionar dilemas éticos o implementar acciones de mejora, actividades propias de la matrona o el matrón. El análisis de casos y el video análisis también son instrumentos de entrenamiento (VázquezMata y Guillamet-Lloveras, 2009). El juego de roles, a diferencia del anterior, quienes actúan son estudiantes que asumen con autonomía el papel de paciente o profesional, ante una situación simulada. “Esta metodología se utiliza para el entrenamiento de múltiples competencias como 
doi: http://dx.doi.org/10.15359/ree.23-2.9

URL: http://www.una.ac.cr/educare

CORREO: educare@una.cr

las habilidades relacionales, análisis de errores por equipos multidisciplinares o para diseñar acciones de mejora de la calidad" (Durá, 2013, p. 76).

A modo de ejemplo, se le asigna a un grupo de cuatro personas una situación clínica relacionada con una gestante que cursa un embarazo de término y acude a un consultorio para ser atendida, por encontrarse con contracciones uterinas frecuentes. Va acompañada por su pareja y personal técnico paramédico que se encarga del traslado de la paciente en silla de ruedas hacia la ambulancia. Cada participante asume el rol asignado y lo caracteriza como tal.

Por medio del juego de roles se desarrollan, preferentemente, las competencias de comunicación, consistencia ética, toma de decisiones y trabajo en equipo y las competencias asistenciales obstétricas relacionadas con la atención integral de una mujer en su periodo reproductivo normal y patológico, que es asistida en forma eficiente ante la emergencia.

El role play permite darse cuenta de un modo racional de los propios fallos y disfunciones al tiempo que genera un "choque vivencial" que lleva al grupo a una toma de contacto con unos modos emocionales propios y hechos visibles en el proceso pedagógico. La persona que "actúa" vive la experiencia metida en la piel de la otra y le lleva, en muchas ocasiones, a una profunda revisión y a un posible cambio personal (Martínez-Riera, Sanjuán-Quiles, Cibanal-Juan y PérezMora, 2011).

\section{Simuladores de paciente completo}

Los simuladores de paciente completo son maniquíes de proporciones similares a una persona, tanto infantil como adulta (Velasco, 2013). Estos modelos tienen la característica de ser robotizados con uso de sistemas informáticos que permiten simular situaciones fisiológicas y patológicas, y conducir problemas críticos que asemejen la realidad. En esta categoría incluimos los modelos obstétricos, Noelle, SimMon, Victoria (Palés y Gomar, 2010). “La simulación reproduce un cuerpo humano completo, con un software que dota al maniquí de todas las funciones cardíacas, vasculares y pulmonares" (Palés y Gomar, 2010, p. 156). Las respuestas vocales pueden ser transmitidas por un micrófono unido al control de una sala, lo cual agrega a más realidad al escenario; incluso, vía internet, puede manejarse el control desde fuera de la sala de simulación (Seropian, Brown, Gavilanes y Driggers, 2004).

Lo anterior, permite que el estudiantado explore al robot, se oriente en la situación clínica e inicie un conjunto de habilidades desde lo básico a lo complejo (Palés y Gomar, 2010). Es decir, con este tipo de simulador, se puede realizar simulación de baja fidelidad para un procedimiento de cuidado básico (cambios posturales, aseos, traslados, etc), simulación de mediana fidelidad para valorar un signo clínico específico (ruidos cardiacos) y simulación de alta fidelidad, en situaciones de crisis, preferentemente. 
Estos simuladores de paciente completo suelen situarse en entornos ambientados como las áreas asistenciales quirúrgicas o de emergencia para enfrentar el estudiantado a un entorno intrahospitalario muy cercano a la realidad. "Otra virtud de este tipo de entrenamiento que no se consigue con otro método docente, es enseñar a los distintos miembros de un equipo asistencial coordinación, liderazgo y comunicación en actuaciones en situaciones críticas, de urgencia o en complicaciones vitales" (Palés y Gomar, 2010, p. 156); inclusive, se puede grabar la escena y luego realizar la retroalimentación o discusión con el grupo participante, a lo que se le llama debriefing. De esta manera, el alumnado observa su actuar y toma de referencia sus errores para eventos futuros simulados o reales.

A modo de ejemplo, en el área obstétrica, se le asigna a un grupo de cuatro personas un caso clínico relacionado con una gestante que cursa un embarazo de término y se encuentra con trabajo de parto. La paciente simulada está hospitalizada en la unidad de urgencia de maternidad con sintomatología normal del periodo de dilatación cervical: contracciones uterinas frecuentes y dolorosas, y latidos cardiacos fetales normales. Está acompañada de su pareja y de un técnico paramédico quien le controla los signos vitales. De pronto, se siente con cefalea, dificultad al mirar, zumbidos en los oídos, dolor en la zona del epigastrio. Transcurren unos segundos e inicia convulsiones con espasmos. La matrona entrega la primera atención ante esta urgencia, que corresponde a mantener vía aérea permeable, solicitar a paramédico que llame al médico de turno y requerir apoyo del resto de integrantes del equipo de salud, diciendo la palabra clave eclampsia (etapa aguda de una hipertensión arterial que puede causar la muerte). Esta escena es más compleja, pues se incorporan una serie de procedimientos como: administración de medicamentos, permeabilización de vía venosa, toma de exámenes, cateterismo vesical para estabilizar la hemodinamia de la paciente, para su posterior cesárea.

A través de este tipo de simulación, se desarrollan las competencias genéricas comunicación, toma de decisiones, consistencia ética y trabajo colaborativo y las competencias asistenciales obstétricas relacionadas con la pesquisa y derivación oportuna de la anormalidad obstétrica en coordinación con el equipo médico, y con el actuar en forma eficiente y eficaz ante situaciones de urgencia durante el proceso reproductivo.

La importancia de la simulación de alta fidelidad no depende de comprar un simulador costoso y de tecnología de punta, sino depende de la capacidad del cuerpo docente para definir las competencias que desean evidenciar a través del entrenamiento y de la evaluación en este tipo de metodología. También, de la calidad en la construcción de escenarios pertinentes, objetivos y definidos respecto al grado de complejidad necesaria para el nivel de sus estudiantes, quienes preferentemente viven estas experiencias simuladas en los dos últimos años de formación de pregrado y con mayor profundidad en el postgrado (Amaya, 2012). 
doi: http://dx.doi.org/10.15359/ree.23-2.9

URL: http://www.una.ac.cr/educare

CORREO: educare@una.cr

\section{Discusiones}

El análisis anterior explicita tres tipos de simulación clínica más usados en el área de obstetricia: entrenamiento con sujetos pacientes simulados (Levine y Swartz, 2008; Palés y Gomar, 2010; Roger, 2017; Vázquez-Mata y Guillamet-Lloveras, 2009), juego de roles o role play (Durá, 2013; Levine y Swartz, 2008; Martínez-Riera et al., 2011; Vázquez-Mata y GuillametLloveras, 2009), y simuladores de paciente completo (Amaya, 2012; Maran y Glavin, 2003; Palés y Gomar, 2010; Seropian et al., 2004; Velasco, 2013).

Iniciamos este análisis discutiendo en forma conjunta el entrenamiento con sujetos pacientes simulados y el juego de roles, dado que tienen similitud en el contexto escénico, tipo de complejidad y fidelidad de simulación clínica, competencias que tributan, y nivel del proceso formativo de la carrera de Obstetricia y Puericultura que se potencian, mayoritariamente. Estos dos tipos de simulación clínica tienen la particularidad de que no emplean ningún objeto como simulador, sino que intervienen sujetos actores o estudiantes que fingen ser pacientes, y ponen en escena una problemática en salud, como por ejemplo realizar un control prenatal en una gestante (Vázquez-Mata y Guillamet-Lloveras, 2009). Su aplicación contribuye al aprendizaje por descubrimiento, al aprendizaje basado en problemas, al aprendizaje significativo que surge de un problema clínico que resolver. Por lo tanto, el medio social, la interacción entre personas y el uso de lenguaje técnico, fortalecen la conducta del matrón y la matrona.

Asimismo, para sustentar la metodología de simulación clínica, hemos tomado de referencia el modelo de Burke enfocado al desempeño personal y organizacional (Burke, 1969). Para este autor, existen factores internos y externos del medio que generan cambio en el comportamiento de las personas y, por ende, en su desempeño individual (Figueroa, Aillon y Salazar, 2013). Este modelo usa cinco elementos como principios generadores que sirven para establecer criterios de organización y selección de sus integrantes: acto, escena, agente, agencia y propósito. El acto es lo que sucedió en el pensamiento o en los hechos; la escena, es el trasfondo del acto, es decir, la situación y el contexto en que ocurrió; el agente es la persona o clase de persona que realizó el acto; los instrumentos que utilizó el agente se denominan agencia, y, finalmente, importa cuál es el propósito que movilizó el acto (Burke, 1969).

Pues bien, podemos afirmar que el modelo de Burke es aplicable a la simulación clínica, porque contiene elementos similares en su didáctica: el acto se refiere a la planificación de la actividad práctica, por la parte docente, y a los conocimientos, habilidades y actitudes del aprendiz; la escena, es la situación clínica en un contexto simulado; el agente es el estudiantado, sujetos pacientes entrenados y docentes; los instrumentos son los simuladores, equipamiento, tecnología y otros recursos, y, finalmente, el propósito se refiere al objetivo de la actividad práctica con el uso de esta metodología. Al finalizar la escena, el personal docente debe realizar la retroalimentación o feed back pertinente, porque en esta etapa se brinda el tiempo para el análisis sistemático del escenario y determinar lo que salió bien y lo que requiere ser mejorado (Dieckmann, 2009). 
También, estos tipos de simulaciones son aplicados en los dos primeros años de formación de pregrado, y no solo potencian las competencias asistenciales obstétricas relacionadas con la atención del proceso reproductivo de la mujer, sino también, las competencias genéricas: interacción social, comunicación, consistencia ética y solución de problemas. Esta última implica una situación en la que se trata de alcanzar alguna meta y se debe hallar los medios para lograrlo (Chi y Glaser, 1985). Esta capacidad resolutiva es destacada en el perfil profesional de la matrona y el matrón, por su autonomía en la toma de decisiones. Por lo tanto, reafirmamos que estos dos métodos de enseñanza, sujetos pacientes simulados y juego de roles, son efectivos en la formación de este grupo profesional, pues permiten desarrollar el aprendizaje significativo en su etapa preclínica.

Ahora bien, el tercer tipo de simulación clínica, los simuladores de paciente completo, cuyo uso emerge en la medicina en el año 1999 (Kohn, Corrigan, Donaldson y CQHCA, 2000), con el fin de otorgar una atención segura, efectiva y centrada en el paciente, sin riesgos de errores humanos y, así, evitar los eventos adversos que llevan a desenlaces desfavorables para pacientes (Sachdeva y Blair, 2004). En la actualidad, gracias a los avances tecnológicos y a la capacitación docente, también se ha insertado esta simulación clínica de alta fidelidad en la formación de obstetricia. Este método emplea el uso de simuladores robotizados de proporciones similares a una persona con el cual se puede simular situaciones fisiológicas y patológicas (Palés y Gomar, 2010), de alta complejidad, como por ejemplo, la atención de un parto vaginal o el enfrentar una crisis eclámptica (crisis hipertensiva severa) de una gestante con trabajo de parto en una unidad de urgencia de maternidad, en cuyo escenario simulado, el estudiantado de obstetricia debe poner a prueba una amplia gama de competencias para proveer atención de calidad a la paciente, como: trabajo en equipo, liderazgo, profesionalismo, destrezas de relación interpersonal, de comunicación y toma de decisiones (Epstein y Hundert, 2002). Según Amaya (2012), la complejidad "es un factor emocional determinante del éxito (aprendizaje), a través de la simulación de alta fidelidad" (p. 48S). Por ende, el estudiantado motivado logrará un aprendizaje significativo, de lo contrario, sentirá decepción de su desempeño.

Pues bien, para comprender el aporte de los simuladores de paciente completo en la formación de las futuras generaciones de matrones y matrones, debemos enfocar nuestra mirada en algunas perspectivas teóricas, que sustentan esta didáctica. Una de ellas es la teoría de Kolb (1984), quien define el aprendizaje como el proceso mediante el cual se crea conocimiento a través de la transformación de la experiencia (p. 38). Para ello, se propone vivir una práctica concreta e inmediata en la que cada participante demuestra sus habilidades y conocimientos para resolver diferentes problemas clínicos críticos. Posteriormente, los participantes reflexionan de manera activa sobre la toma de decisiones y la resolución del problema vivido; integran sus meditaciones y forman un modelo teórico, que permite poner a prueba los conceptos incorporados en nuevos escenarios simulados controlados o en el entorno 
doi: http://dx.doi.org/10.15359/ree.23-2.9

URL: http://www.una.ac.cr/educare

CORREO: educare@una.cr

clínico real (Davidson, 2008). A esto se suma la perspectiva vygotskiana, desde la importancia de las funciones mentales y acción del ser humano, que se construyen a través de las relaciones entre los individuos y la importancia del medio (Wertsch, 1993).

También, los escenarios de simulación clínica de alta complejidad se sustentan teóricamente en el modelo circunflejo de Russell, el cual se relaciona con el estímulo emocional y la predominancia de los elementos de placer y activación (Amaya, 2012). Si se mantiene al estudiantado entre los ejes de actividad placentera predominante, es factible lograr un aprendizaje significativo y duradero en el tiempo. Por ello, esta metodología se emplea en estudiantes de cursos avanzados, porque deben equilibrar las emociones con el saber hacer y el actuar, a fin de demostrar una conducta profesional idónea ante una situación de riesgo vital.

Consecuentemente, estos simuladores integrados son un recurso didáctico porque poseen características humanas, como estatura, parámetros vitales (cardiovasculares y respiratorios, principalmente) y altavoces para comunicarse por redes inalámbricas con los participantes de la escena, que potencian el realismo en situaciones críticas. Para comprender su aporte en el aprendizaje, debemos integrarlo con la neurociencia cognitiva, en la que el observador trata de entender los estados mentales de otros seres, creando una copia de ellos en su propia mente. El ser humano expresa su pensar y su sentir a través de sus actos, gestos y lenguaje que son percibidos por las neuronas de espejo de otros sujetos observadores (Coderch, 2009). Por ende, "el cerebro del obsevador reproduce, gracias al sistema en espejo, aquello que está observando -un acto motor, una expresión facial, un tono de voz que manifiesta una determinada emoción, etc.- estableciéndose asi una línea directa de comunicación con el sujeto observado" (Coderch, 2009, p. 49). En este caso, el cerebro del estudiantado reproduce la voz y el movimiento del simulador, como si fuese una gestante que está cursando su parto o una situación de urgencia obstétrica, que requiere de su atención especializada.

Además, podemos mencionar que en este tipo de simulación clínica se aplica la percepción háptica, que es la combinación táctil con la kinésica. Según Ballesteros (1993), la háptica corresponde a "percibir los objetos ... [del] entorno cuando [se utiliza] el sentido del tacto de una manera propositiva, esto es, de forma activa y voluntaria" (p. 313). Por lo tanto, este tipo de percepción permite movimientos exploratorios, los que tienen un valor significativo en el desarrollo de habilidades táctiles con el simulador de paciente completo. Como, por ejemplo, al realizar las maniobras de extracción fetal en un período expulsivo, o técnicas básicas como punción venosa, toma de exámenes, administración de medicamentos, entre otras.

Por ende, el estudiantado interactúa con el simulador, se comunica, desarrolla procedimientos, reflexiona, toma decisiones y, en definitiva, actúa con la mentalidad que es una paciente en situación de riesgo, y que es su deber salvar la vida de la madre y su hijo. También desarrolla el trabajo colaborativo, que fomenta el pensamiento crítico y la resolución 
de problemas desde un enfoque constructivista. Por lo tanto, afirmamos que la simulación clínica de alta fidelidad es una de las mejores herramientas para la práctica de las competencias genéricas y específicas de la obstetricia, necesarias para un buen desempeño laboral. Además, a través de esta metodología,"cobra importancia la reflexión del alumno sobre su propio proceder cognitivo y procedimental, además de reconocer los procesos metacognitivos que despliega al aprender" (Piña-Jiménez y Amador-Aguilar, 2015, p. 157).

El éxito de esta simulación depende de la existencia de una alta fidelidad física, en la cual se logren desarrollar habilidades manuales, una alta fidelidad conceptual en la cual se desarrolla el razonamiento clínico y la habilidad para solucionar problemas y, por último, la alta fidelidad emocional o vivencial en la cual se favorece la retención de información mediante el manejo de procesos complejos que involucran conocimientos o emociones, su aplicación resulta más favorable en los últimos años de formación de pregrado y, se profundiza en el postgrado. Sin embargo, a pesar de que el realismo en este tipo de simulación aproxima la teoría a la práctica, no reemplaza el campo clínico intrahospitalario.

Definitivamente, hemos revisado tres tipos de simulaciones que se desarrollan en la formación de este grupo profesional. Claramente, cada uno de ellos conduce a objetivos y enfoques de aprendizajes acordes con el currículo de esta carrera.

Consideramos dentro de sus ventajas: 1) Aportan significativamente en el aprendizaje al complementar la enseñanza tradicional. 2) Dan la posibilidad de repetir la técnica las veces que sea necesario. 3) El estudiantado aprende del error y construye un nuevo aprendizaje. 4) Ofrecen escenarios planificados y protegidos, de lo más simple como una sala de atención para realizar una entrevista obstétrica, a lo más complejo, como una unidad de pabellón de maternidad para atender una paciente en parto. 5) Permiten el feed back o debriefing en tiempo real, donde el alumnado puede reconocer sus errores, reflexionar sobre estos y corregir los fallos clínicos y de coordinación (López, Ramos, Pato y López, 2013).

Planteamos como desafíos: 1) Personal docente capacitado con esta didáctica, que permita modificar el modelo educativo academicista. 2) Currículo renovado con inclusión de preparación preclínica antes del ingreso al campo real. 3) Disposición de medios económicos, técnicos e infraestructura para implementar la simulación de alta fidelidad. 4) Oferta de cursos de perfeccionamiento académico que potencien la educación continua.

Como conclusión, la simulación clínica en el área de obstetricia aporta significativamente en la enseñanza y aprendizaje de la matrona y del matrón en formación. Los diversos tipos de simulación de baja, mediana y alta fidelidad seleccionados potencian el aprendizaje por descubrimiento, basado en problemas, experiencial y significativo, así como también, poseen dimensiones organizacionales y de autorregulación ante los estímulos que son sustentados en los modelos de Burke y de Russell, lo que se traduce en su complejidad y fidelidad cercana al 
doi: http://dx.doi.org/10.15359/ree.23-2.9

URL: http://www.una.ac.cr/educare

CORREO: educare@una.cr

realismo. Por ende, el entorno, los sentidos, el lenguaje, la relaciones entre individuos interactúan entre sí, acercándose a los contextos clínicos intrahospitalarios. Esto ha permitido mejorar la seguridad y confianza del estudiantado en las carreras de Obstetricia y Puericultura del país, al enfrentar sus prácticas profesionales con pacientes reales. Sin embargo, existen escasos estudios que evidencien el desarrollo de estas metodologías en la etapa preclínica en la matronería. Por lo tanto, se hace necesario desarrollar programas de formación de simulación clínica dirigidos a matronas y matrones docentes, que permita potenciar esta técnica en el pregrado como en el postgrado, dada la importancia que tiene esta herramienta activa para el proceso formativo, así como también, evidenciar su aplicación en investigaciones educativas. Además, sería de gran utilidad que todas las carreras de obstetricia y puericultura conformen una red como centros de formación en simulación clínica profesionalizante, reconocida y enmarcada en las políticas institucionales de cada universidad. De esa manera, se apoyaría la integración formal de esta nueva metodología de enseñanza al currículo, lo que implicaría un cambio de cultura formadora para las generaciones futuras.

Finalmente, la matronería es la ciencia y la profesión que se encarga de la salud reproductiva y salud sexual de la mujer a lo largo de toda su vida. El aprendizaje adquirido durante los años de formación permite entregar una atención integral y de calidad a las personas. Gracias a esta técnica, existe un menor número de eventos adversos desfavorables para las pacientes. Por lo tanto, podemos concluir que la simulación clínica es una herramienta necesaria para el aprendizaje de la matrona y del matrón.

\section{Referencias}

Alcolea-Cosín, M. T., Oter-Quintana, C., Martínez-Ortega, R. M., Sebastián-Viana, T. y PedrazMarcos, A. (2012). Aprendizaje basado en problemas en la formación de estudiantes de enfermería: Impacto en la práctica clínica. Educación Médica, 15(1), 23-30. Recuperado de http://scielo.isciii.es/scielo.php?script=sci arttext\&pid=S1575-18132012000100007

Altamirano, J. (2011). Innovación curricular en el dominio asistencial. Revista Electrónica de Desarrollo de Competencias (REDEC), 1(7), 77-100.

Amaya, A. (2012). Simulación clínica y aprendizaje emocional. Revista Colombiana de Psiquiatría, 41, 44S-51S. Recuperado de http://www.scielo.org.co/pdf/rcp/v41s1/v41s1a06.pdf

Argudín, Y. (2001). Educación basada en competencias. Educar. Revista de educación, 16, 1-15. Recuperado de http://www.lie.upn.mx/docs/docinteres/Educacion basada en competencias.doc 
Arriazu, R. (2013). La adaptación del plan Bolonia en las aulas: Una perspectiva histórica y crítica para entender las claves y estrategias de las universidades española. Barcelona: Octaedro. Recuperado de https://edicionesmagina.com/appl/botiga/client/img/16057.pdf

Ausubel, D. P., Novak, J. D. y Hanesion, H. (1990). Psicología educativa. México: Trillas.

Ballesteros, S. (1993). Percepción háptica de objetos y patrones realzados: Una revisión. Revista Psicothema, 5(2), 311-321. Recuperado de http://www.redalyc.org/pdf/727/72705209.pdf

Battoccio, G. (2016). Escuela de Obstreticia y Puericultura inauguró una moderna sala de simulación clínica con fines docentes. Revista Contacto, 4(9), 3. Recuperado de https:// obstetricia.uv.cl/inicio/wp-content/uploads/2017/05/BoletinContactoN9.pdf

Beneitone, P., Esquetini, C., González, J., Marty, M., Siufi, G. y Wagenaar, R. (Eds.). (2007). Reflexiones y perspectivas de la educación superior en América Latina (Informe final -Proyecto TuningAmérica Latina 2004-2007). Bilbao: Universidad de Deusto. Recuperado de http:// tuningacademy.org/wp-content/uploads/2014/02/TuningLAllI Final-Report SP.pdf

Bravo, C. (15 de noviembre de 2013). Académicos de la Facultad de Ciencias de la Salud se capacitaron con clases de simulación clínica. PrensaUA. Recuperado de http://www. comunicacionesua.cl/2013/11/15/academicos-de-la-facultad-de-ciencias-de-la-salud-secapacitaron-con-clases-de-simulacion-clinica/

Bruner, J. S. (1961). The Act of Discovery. Harvard Educational Review. 4: 21-32

Bruner, J. S. (1966). Toward a Theory of Instruction. Cambridge, MA: Harvard University Press

Burke, K. (1969). A grammar of motives. Berkeley: University of California Press.

Camargo-Escobar, I. M. y Pardo-Adames, C. (2008). Competencias docentes de profesores de pregrado: Diseño y validación de un instrumento de evaluación. Revista Universitas Psychologica, 7(2), 441-455. Recuperado de http://www.redalyc.org/articulo. oa?id=64770211

Centro de Habilidades Clínicas (CHC). (2017). Facultad de Medicina de la Universidad de Chile. Recuperado de http://chc.med.uchile.cl/

Chi, M. y Glaser, R. (1985). Problem-solving ability. In R.J. Sternberg (Ed.), Human abilities: An information-processing approach (pp. 227-250). New York: Freeman. Recuperado de https://files.eric.ed.gov/fulltext/ED257630.pdf

Coderch, J. (2009). Neurociencia y modelo relacional. Clínica e Investigación Relacional, 3(1), 39-53. Recuperado de http://www.psicoterapiarelacional.es/Portals/0/Documentacion/ JCoderch/Coderch 2009 Neurociencia-Modelo-Relacional CelR V3N1.pdf 
doi: http://dx.doi.org/10.15359/ree.23-2.9

URL: http://www.una.ac.cr/educare

CORREO: educare@una.cr

Comisión Nacional de Acreditación (CNA). (2007). Criterios de evaluación para la acreditación de carreras de Obstetricia y Puericultura. Recuperado de http://www.acreditadoradechile.cl/ wp-content/uploads/2011/11/Obstetricia.pdf

Davidson, M. (2008). The taxonomy of learning. International Anesthesiology Clinics, 46(4), 1-15. doi: https://doi.org/10.1097/AIA.0b013e318187691c

Delors, J. (Preside). (1996.). Los cuatro pilares de la educación. En J. Delors (Preside), Informe a la UNESCO de la Comisión internacional sobre la educación para el siglo XXI. La educación encierra un tesoro (pp. 91-103). Madrid: Santillana Ediciones Unesco. Recuperado de http:// uom.uib.cat/digitalAssets/221/221918 9.pdf

Dewey, J. (2004). La educación tradicional frente a la educación progresista. En J. Dewey (Autor), Experiencia y educación (pp. 65-126). Madrid: Biblioteca Nueva Recuperado de https:// tecnoeducativas.files.wordpress.com/2015/08/dewey-experiencia-y-educacion.pdf

Díaz-Barriga, F. (2005). Desarrollo del currículo e innovación: Modelos e investigación en los noventa. Revista Perfiles Educativos, 27(107), 57-84. Recuperado de http://www.scielo.org. $\underline{\mathrm{mx} / \mathrm{pdf} / \text { peredu/v27n107/n107a04.pdf }}$

Dieckmann, P. (2009). Simulation is more than technology - the simulation setting. Recuperado de http://www.laerdaltraining.com/sun/enable/PDF/dieckman article.pdf

Durá, M. J. (2013). La simulación clínica como metodología de aprendizaje y adquisición de competencias en enfermería (Memoria doctoral). Universidad Complutense de Madrid, España. Recuperado de https://metodoinvestigacion.files.wordpress.com/2014/11/ simuacic3b3n-clc3adnica-como-metodologc3ada-de-aprendizaje-u-complutense.pdf

Eleizalde, M., Parra, N., Palomino, C., Reyna, A., \& Trujillo, I. (2010). Aprendizaje por descubrimiento y su eficacia en la enseñanza de la biotecnología. Revista de Investigación, 34(71), 271-290. Recuperado de http://www.scielo.org.ve/scielo.php?pid=S1010$\underline{29142010000300014 \& \text { script }=\text { sci arttext } \& \text { tlng }=p t}$

Epstein, R. M y Hundert, E. M. (2002). Defining and assessing professional competence. JAMA. Journal of the American Medical Association, 287(2), 226-235. doi: https://doi.org/10.1001/ jama.287.2.226

Escudero, E. X., Fuentes, C M., González, M. J. O. y Corvetto, M. A. (2016). Simulación en educación para ciencias de la salud: ¿Qué calidad hemos alcanzado en Chile? ARS MEDICA Revista de Ciencias Médicas, 41(3), 16-20. doi: http://dx.doi.org/10.11565/arsmed.v41i3.394

Feldman, R. S. (2005). Psicología: Con aplicaciones en países de habla hispana (6a ed.). México: McGraw-Hill. 
Figueroa, B. E., Aillon, M. y Salazar, O. (2013). La acción mediada, una unidad de análisis para revisar las prácticas de lectura y escritura hipertextual en la formación de profesores. Revista de Universidad y Sociedad del Conocimiento. RUSC, 10(1), 75-88. doi: http://dx.doi. org/10.7238/rusc.v10i1.1291

Gaba, D. M. (2004). The future vision of simulation in health care. Qual Saf Health Care, 13(1), i2i10. doi: https://doi.org/10.1136/qhc.13.suppl 1.i2

Hawes, G. y Corvalán, O. (2005). Construcción de un perfil profesional. Chile: Instituto de Investigación y Desarrollo Educacional. Universidad de Talca.

Kohn L. T., Corrigan J. M., Donaldson M. S. y Committee on Quality of Health Care in America, Institute of Medicine. (Eds.). (2000). To err is human: Building a safer health system. Washington D.C.: Institute of Medicine, National Academies Press. Recuperado de https:// www.ncbi.nlm.nih.gov/pubmed/25077248

Kolb, D. A. (1984). Experiential learning: Experience as the source of learning and development. Englewood Cliffs, NJ: Prentice Hall.

Lane, J. L., Slavin, S., Ziv, A. (2001). Simulation in medical education: A review. Revista Simulation \& Gaming, 32(3), 297-314. doi: https://doi.org/10.1177/104687810103200302

Levine, A. I., y Swartz M. H. (2008). Standardized patients: The "other" simulation. Journal of Critical Care, 23(2), 179-184. doi: https://doi.org/10.1016/j.jcrc.2007.12.001

López, M., Ramos, L., Pato, O. y López S. (2013). La simulación clínica como herramienta de aprendizaje. Cma. Cirugía mayor ambulatoria, 18(1), 27-31. Recuperado de http://www. asecma.org/Documentos/Articulos/05 181 FC Lo\%C2\%A6\%C3\%BCpez.pdf

Maran, N. J., y Glavin, R. J. (2003). Low- to high-fidelity simulation - a continuum of medical education? Medical Education, 37(1), 22-28. doi: https://doi.org/10.1046/j.1365-2923.37.s1.9.x

Martínez-Riera, J. R., Sanjuán-Quiles, Á., Cibanal-Juan, L., Pérez-Mora, M. J. (2011). Roleplaying en el proceso de enseñanza-aprendizaje de enfermería: Valoración de los profesores. Cogitare Enfermagem, 16(3), 411-417. Recuperado de https://rua.ua.es/dspace/ bitstream/10045/24306/1/2011 Martinez Riera etal Cogitare Enferm.pdf

Ortega, R. (2008). Competencias para una educación cosmopolita. Andalucía Educativa, 66. Recuperado de http://redined.mecd.gob.es/xmlui/bitstream/ handle/11162/264/00120123000119.pdf?sequence=1\&isAllowed $=y$

Palés, J. L. y Gomar, C. (2010). El uso de las simulaciones en educación médica. Teoría de la educación: Educación y cultura en la sociedad de la información, 11(2), 147-169. Recuperado de $\quad$ http://www.ub.edu/medicina unitateducaciomedica/documentos/Lus\%20de\%20 les\%20simulacions\%20en\%20educacio\%20medica.pdf 
doi: http://dx.doi.org/10.15359/ree.23-2.9

URL: http://www.una.ac.cr/educare

CORREO: educare@una.cr

Panorama Universidad de Concepción (UDEC). (2016). Obstetricia y puericultura UdeC inaugura remodelación de dependencias y pabellones de simulación. Recuperado de http:// www.udec.cl/panoramaweb2016/content/obstetricia-y-puericultura-udec-inauguraremodelaci\%C3\%B3n-de-dependencias-y-pabellones-de

Piña-Jiménez, I. y Amador-Aguilar, R. (2015). La enseñanza de la enfermería con simuladores, consideraciones teórico-pedagógicas para perfilar un modelo didáctico. Revista Enfermería Universitaria, 12(3), 152-159. doi: http://dx.doi.org/10.1016/j.reu.2015.04.007

Ramírez, L. V. y Medina, M. G. (2008). Educación basada en competencias y el proyecto Tuning en Europa y Latinoamérica Su impacto en México. Ide@s CONCYTEG, 3(39), 97-114. Recuperado de http://mibibliotecatec.weebly.com/uploads/5/4/5/7/54577939/edu basada competencias proyecto tuning.pdf

Riesco, M. (2008). El enfoque por competencias en el EEES y sus implicaciones en la enseñanza y el aprendizaje. Revista Tendencias Pedagógicas, 13, 79-105. Recuperado de https://revistas. uam.es/tendenciaspedagogicas/article/view/1892

Rodríguez, H. (2007). El paradigma de las competencias hacia la educación superior. Revista Facultad de Ciencias Económicas, 15(1), 145-165. Recuperado de http://www.redalyc.org/ articulo.oa?id=90915108

Rodríguez, J. (2002). La evaluación en la universidad. La evaluación del aprendizaje de los alumnos universitarios. En C. Mayor (Coord.), Enseñanza y aprendizaje en la educación superior (pp. 85-120). Barcelona: Octaedro.

Roger (2017). Los pacientes simulados en la evaluación de la competencia. Boletín Doctutor de Educación Médica. Recuperado de http://www.doctutor.es/2017/04/12/los-pacientessimulados-en-la-evaluacion-de-la-competencia/

Sachdeva, A. K. y Blair, P. G. (2004). Educating surgery residents in patient safety. Surgical Clinics of North América, 84(6), 1669-1698. doi: https://doi.org/10.1016/j.suc.2004.06.004

Schunk, D. H. (2005). Teorías del aprendizaje. México: Pearson Educación.

Seropian, M. A., Brown, K., Gavilanes, J. S. y Driggers, B. (2004). Simulation: Not just a manikin. Journal of Nursing Education, 43(4), 164-169. doi: https://doi.org/10.3928/0148483420040401-04

Universidad de Atacama (UDA). (2015). Obstetricia y puericultura UDA adquirió seis simuladores clínicos de alta tecnología. Recuperado de http://www.uda.cl/index.php?option=com co ntent\&view=article\&id=1775:obstetricia-y-puericultura-uda-adquirio-seis-simuladoresclinicos-de-alta-tecnologia\&catid=15\&ltemid=277 
Universidad de Las Américas (UDLA). (2017). Ficha N. ${ }^{\circ}$ 16. Dramatizaciones, simulaciones o role play. Procedimientos de evaluación auténtica. Procedimientos evaluativos utilizados en el aula. Recuperado de http://www.udla.cl/portales/tp9e00af339c16/uploadlmg/File/ fichas/Ficha-16-dramatizaciones.pdf

Universidad de La Frontera (UFRO). (2012). Experiencias de formación en el pregrado. Recuperado de http://webcache.googleusercontent.com/search?q=cache:0vHneOn5dDwJ:pregrado. ufro.cl/index.php/component/docman/doc download/4-libro-2014\%3Fltemid\%3D+\&c $\mathrm{d}=1 \& \mathrm{hl}=\mathrm{es} \& \mathrm{ct}=\mathrm{clnk} \& \mathrm{gl}=\mathrm{cr}$

Universidad de Tarapacá (UTA). (2017) Capacitación a académicos de la FACSAL en Centro de Simulación Clínica. Recuperado de http://www.uta.cl/capacitacion-a-academicos-de-lafacsal-en-centro-de-simulacion-clinica/web/2017-01-27/164533.html

Urra, E., Sandoval, S., e Irribarren, F. (2017). El desafío y futuro de la simulación como estrategia de enseñanza en enfermería. Revista Investigación en Educación Médica, 6(22), 119-125. Recuperado de http://www.scielo.org.mx/scielo.php?pid=\$200750572017000200009\&script $=$ sci arttext

Vázquez-Mata, G. y Guillamet-Lloveras, A. (2009). El entrenamiento basado en la simulación como innovación imprescindible en la formación médica. EDUC MED, 12(3), 149-155. doi: https://doi.org/10.4321/S1575-18132009000400004

Velasco, A. (2013). Simulación clínica y enfermería, creando un ambiente de simulación (Trabajo de grado). Universidad de Cantabria, España. Recuperado de https://metodoinvestigacion. files.wordpress.com/2014/11/simulacic3b3n-cc3b1inica-y-efermerc3ada-creando-unambiente-de-simulacic3b3n-u-de-cantabria.pdf

Wertsch J. V. (1993). Voces de la mente. Un enfoque sociocultural para el estudio de la acción mediada. Madrid: Visor.

Yániz, C. y Villardón, L. (2006). Planificar desde competencias para promover el aprendizaje. Bilbao: Mensajero.

Ziv, A., Wolpe, P. R., Small, S. D. y Glick, S. (2003). Simulation-based medical education: An ethical imperative. Revista Academic Medicine, 78(8), 783-788. Doi: https://doi. org/10.1097/00001888-200308000-00006 